\title{
Changes in rib cage geometry during childhood
}

\author{
P OPENSHAW, S EDWARDS, P HELMS
}

From Guy's Hospital, St Bartholomew's Hospital, and Brompton Hospital, London

\begin{abstract}
Age related changes in rib cage geometry were found from measurements made on chest radiographs from 38 individuals aged from 1 month to 31 years and on computed tomography (CT) scans in another 28 individuals, aged from 3 months to 18 years. Chest radiographs were taken for minor respiratory symptoms or fever and only films showing no abnormality were used. CT scans were obtained in children undergoing staging for solid tumours in whom no intrathoracic deposits were found. In infants and very young children the ribs were found to be more horizontal and the sternal clavicular heads and diaphragmatic domes higher than in older children and young adults. Most of these changes were observed in the first two years of life, with something close to the adult pattern by the age of 2 years. Similarly cross sectional chest shape changed from the rounded infantile form to the more ovoid adult form by the same age. The configuration of the ribs observed in infancy and early childhood reduces the potential for thoracic expansion and may contribute to the frequency of respiratory problems found in the very young.
\end{abstract}

Respiratory problems are frequent in childhood and most significant illnesses in preschool children are referrable to the respiratory tract. ${ }^{1}$ The reasons for this age distribution are likely to be the immaturity of the host defences in the lungs and the lesser ability of the respiratory pump to cope with the increased respiratory loads associated with airflow obstruction. The respiratory pump (rib cage, accessory muscles, diaphragm, and abdominal muscles) is thought to be less efficient in the very young child than in the mature adult because of the instability of the thoracic cage and the lower efficiency of the diaphragm. ${ }^{2}$ Not only is the rib cage more pliable in the very young ${ }^{3}$ but the ribs also appear to be more horizontal than in the adult. ${ }^{4-6}$ The pliable rib cage allows the chest wall to move inwards during strong diaphragmatic contraction, while the more horizontal lie of the ribs is likely to limit the potential for thoracic expansion by rib cage movement in the cephalad direction.

Although it is known that rib cage geometry changes from early infancy to adulthood, the timing of this change has not be described.

Address for reprint requests: Dr P Openshaw, Department of Respiratory Medicine, Hammersmith Hospital, London W12 0HS.

Accepted 17 April 1984

\section{Methods}

We examined radiographs in 38 subjects aged 1 month to 31 years and computed tomography (CT) scans of the thorax in 28 children and adolescents aged 3 months to 18 years.

\section{CHEST RADIOGRAPHS}

Chest radiographs were taken from the paediatric accident and emergency department at Guy's Hospital and from adults having routine films taken before employment at the Brompton Hospital. Radiographs which were poorly orientated, as assessed by the position of the sternal heads of the clavicles in relation to the spine, were excluded, as were films from any subject with a history of wheezing, asthma, or recent pneumonia. All of the children underwent radiographic examination because of minor respiratory symptoms or fever, and only those films showing no abnormality were accepted for the study. Clear celluloid film was placed over each radiograph and the following points were marked: the most lateral points of ribs 1-10; the necks of ribs 1-10; the centres of each vertebral body; the dome of each diaphragm; the position of the clavicles. For each film a "slope index" was calculated for each rib pair. The maximum potential width of each rib pair was calculated by adding the distance between the necks and most lateral points of the ribs to the dis- 
tance between the necks. The separation of the most lateral points of each rib pair was then measured directly and expressed as a ratio of the maximal potential width. Once a slope index had been calculated for each rib pair an average was taken for ribs 7-10.

In infants and very young children radiographs were taken with the film behind the chest whereas older children and adults were positioned with the film in front of the chest. A further difference was that films taken in very young uncooperative children were taken in the upright posture with the arms partly supported. To observe how these different techniques could affect our measurements we compared films taken posteroanterior and anteroposterior in 22 children and adults above the age of 5 years and films taken with arm support in the erect posture and without arm support in the supine posture in infants of less than 6 months.

\section{CT SCANS}

CT scans were obtained from children who were being examined to stage lymphomas and other solid tumours. Only scans from those children in whom no deposits were found in the thoracic cavity were used in this analysis.

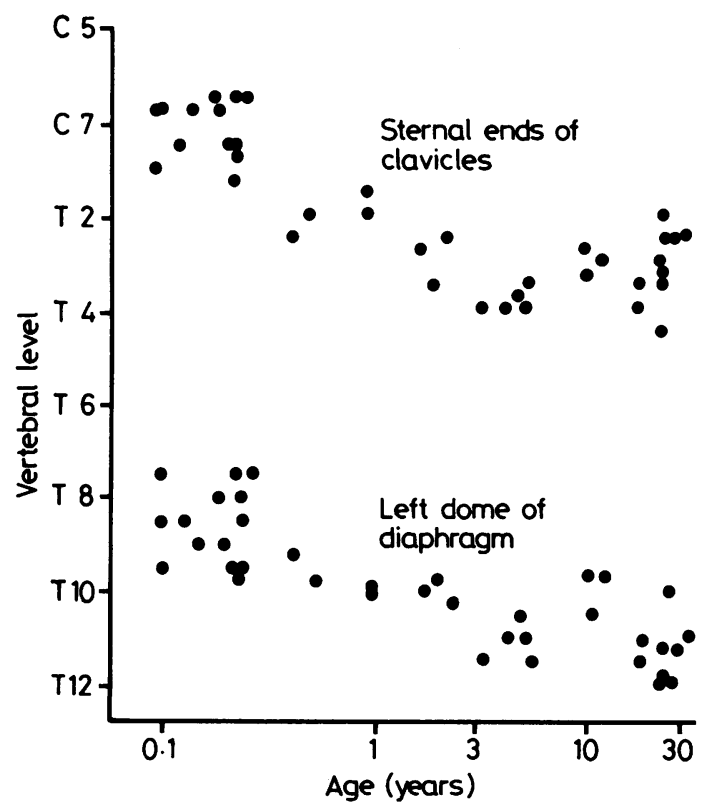

Fig 1 Vertebral level of projection of sternal clavicular heads and the dome of the left hemidiaphragm based on chest radiographs, showing the descent with increasing age (measurements taken from the centre of each vertebral body).
From the CT scans the thoracic index ${ }^{7-11}$ was derived by dividing the anteroposterior diameter by the lateral diameter at three thoracic levelsnamely, the manubriosternal junction, the diaphragmatic dome, and midway between these two reference levels. The thoracic index used was the mean of that found at these three levels.

\section{Results}

A similar pattern of changing rib cage geometry emerged from both chest radiograph and CT scan measurements. For diaphragm level, sternal head level, rib slope index, and thoracic index the most rapid period of change appeared to be from birth to the age of about 2 years (figs 1 and 2). The mean position of the left diaphragmatic dome was found to be at the level of the 8th thoracic vertebra at birth, descending to thoracic vertebra 11 in the young adult. For subjects of all ages the right diaphragmatic dome was found to be a half vertebral space higher than the left. The medial ends of the clavicles also changed their projection from between cervical vertebra 7 and thoracic vertebra 1 at birth to the body of cervical vertebra 3 in the adult subjects. The rib slope index decreased from birth to adulthood, a change which was complete by 10 years of age. The cross sectional thoracic index also changed over this period, with a more rounded shape in early infancy and the establishment of the

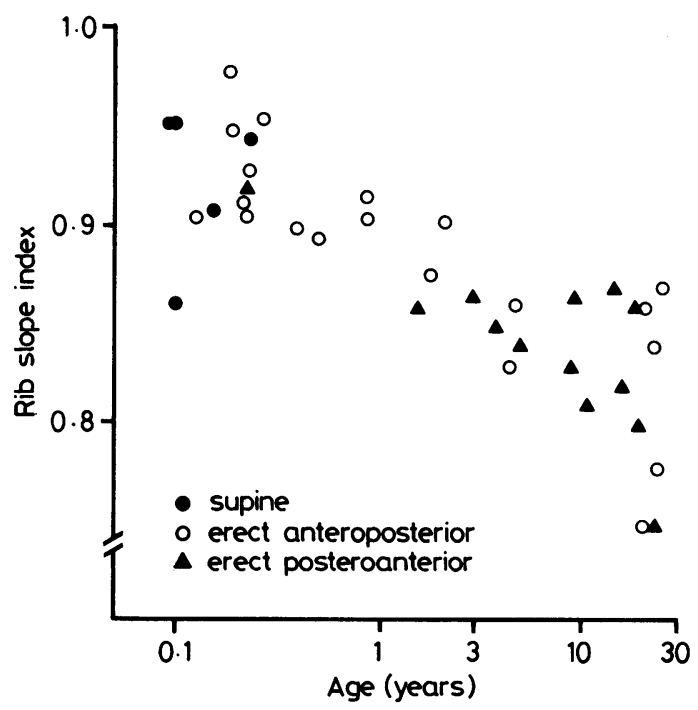

Fig 2 Relationship between the mean slope index for rib pairs 7-10 and age based on chest radiographs. A slope index of 1 indicates a horizontal lie of the ribs and lower indices a downward slope. 


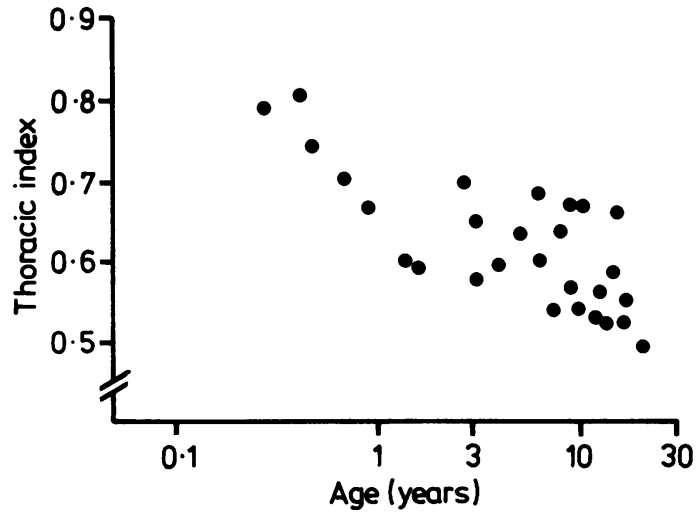

Fig 3 Relationship of the thoracic index (anteroposterior/lateral thoracic diameter) to age based on computed tomography scans.

move ovoid adult pattern by the age of 2-3 years (fig 3).

No significant differences in slope index were found between anteroposterior and posteroanterior films in 22 age matched subjects above the age of 5 years (fig 2) ( $p>0 \cdot 1$, two tailed Mann-Witney $U$ test). In 14 infants less than 6 months of age there was no significant difference between slope indices determined from supine and from erect films (fig 2; $p>0 \cdot 1$, two tailed Mann-Witney U test).

\section{Discussion}

Before we accept the principal findings there are several potential problems with the chest radiograph measurements which must be answered. Firstly, more of the younger children had their radiographs taken with the film behind the chest, whereas older children were usually positioned with the film in front of the chest. No significant differences in slope index, however, were found between anteroposterior and posteroanterior films in subjects more than five years old and this factor seems not to have affected our results. A second systematic error could have arisen from the way very young children are partly supported by their arms to ensure an upright position. If lifting of the arms also lifted the lateral parts of the ribs the slope index taken in subjects of a similar age in the supine posture would be expected to be lower. In infants less than 6 months of age no such effect was observed. Young children are unlikely to achieve degrees of lung inflation similar to those produced by older, more mature subjects since inspiration is timed from the intake of breath before the child cries. The change in rib slope index is unlikely to be due to incomplete inspiration because in this case the changes with age would be
Thoracic configuration

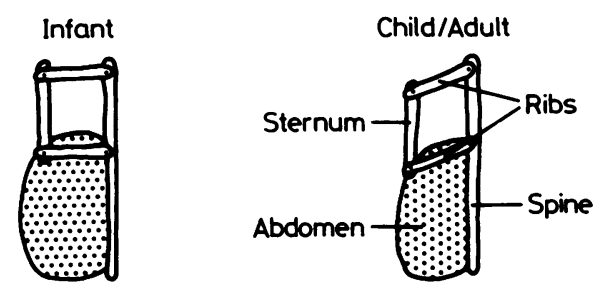

Thoracic cross section
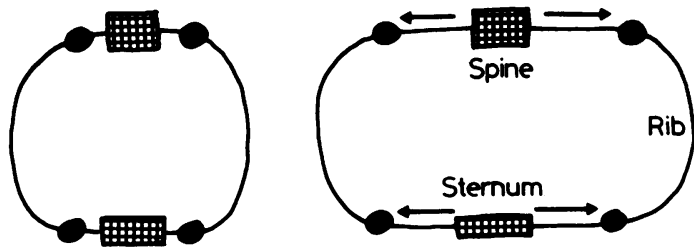

Fig 4 Observed changes in configuration and cross sectional shape of the thorax from infancy to early childhood. Upper panel: infantile and adult rib cage configurations. Lower panel: how rib growth at costochondral junctions and posterior rib angles could explain the observed changes in cross sectional shape of the thorax.

opposite to those actually observed. Finally, the change in clavicular and diaphragmatic levels would be expected if the young child systematically adopted a lordotic posture when held erect; the similar features seen with the supine views (fig 2), however, suggest that this is unlikely.

The changes in thoracic cross sections of CT scans were similar to changes of external thoracic dimensions found in previous studies. ${ }^{7-11}$ The present study and most of the previous studies were performed at full lung inflation; Howatt and De Muth, ${ }^{8}$ however, reached the same conclusions from measurements made at the resting end tidal phase of respiration, and it has been shown that in normal adult subjects cross sectional chest shape, assessed by computed tomography, appears to maintain a constant form over the vital capacity range. ${ }^{12}$

Accepting that the changes we have observed are real, we may speculate why they occur. Engel ${ }^{4}$ ascribes the horizontal lie of the ribs in the very young child to an excess of lung tissue relative to the size of the thoracic cage and states that "the chest grows more rapidly than the lung in late infancy and early childhood." Reduced lung recoil in infancy may partly explain the horizontal lie of the ribsjust as adults with pulmonary emphysema have a barrel chest deformity. ${ }^{13}$ Reduced lung recoil seems improbable, however, as the whole explanation since the diaphragms of young children are higher than in older, more mature individuals (fig 1). 
Another possible explanation is that changes in posture may lead to changes in chest wall shape. In support of this suggestion Krahl cites the case of a congenitally bipedal goat which developed an upright posture. ${ }^{14} \mathrm{~A}$ normal goat's chest is flattened laterally but this goat developed a chest wider than it was deep, similar to that of a human adult.

The timing of the changes in rib slope and the height of the clavicles and diaphragm suggests that posture has an important influence on thoracic shape. These rapid changes from birth to 2 years occur just at the time when the upright posture is being adopted and when the weight of the lungs and abdominal contents could affect the configuration of the thorax. With time the gravitational forces operating on the rib cage together with the effects of rib growth would be expected to result in the observed changes (fig 4).

Whatever the reason for the changes in thoracic shape the very young child would appear to have less potential for thoracic expansion than the older child and adult. Possibly this relative mechanical inefficiency of the rib cage contributes to the frequency of respiratory problems in young children.

We thank Miss $\mathrm{H}$ Whippy for secretarial skills.

\section{References}

' Williams HE, Phelan PD. The epidemiology of acute respiratory infections. In: Respiratory illness in children. Oxford: Blackwell Scientific Publications, 1975.

${ }^{2}$ Muller NL, Bryan AC. Chest wall mechanics and respiratory muscles in infants. Pediatr Clin North Am 1979;26:503-16.

${ }^{3}$ Richards CC, Bachman L. Lung and chest wall compliance of apneic paralyzed infants. J Clin Invest 1961;40:273-8.

${ }^{4}$ Engel S. The child's lung: developmental anatomy, physiology and pathology. London: Edward Arnold, 1947.

${ }^{5}$ Meinert E. Uber topographische Altersveranderungen des Atmungsaparates. Jena: Fischer, 1901.

${ }^{6}$ Howard PJ, Bauer AR. Irregularities of breathing in the newborn period. Am J Dis Child 1949;77:592-609.

' Davenport CB. Thoracic index. Hum Biol 1934;6:1-8.

${ }^{8}$ Howatt WF, De Muth GR. Growth of lung function III. Configuration of the chest. Pediatrics 1965;35:17784.

${ }^{9}$ Meredith HV, Knoth VB. Changes in body proportions during infancy and the pre-school years. I. The thoracic index. Child Develop 1937;8:173-7.

${ }^{10}$ Takohashi H, Absumi H. Age differences in thoracic form as indicated by thoracic index. Hum Biol 1955;27:65-71.

"Scammon RE. Studies on the growth and structure of the infant thorax. Radiology 1927;9:89-95.

12 Pierce RJ, Brown DJ, Holmes M, Cumming G, Denison DM. Estimation of lung volumes from chest radiographs using shape information. Thorax 1979; 34:726-33.

${ }^{13}$ Pierce JA, Ebert RV. The barrel deformity of the chest, the senile lung and obstructive pulmonary emphysema. Am J Med 1958;25:13-22.

${ }^{14} \mathrm{Krahl}$ VE. Anatomy of the mammalian lung. In: Fenn WO, Rahn H, eds. Handbook of physiology. Section 3, vol 1. Baltimore: Waverly Press, 1964:213-84. 